\title{
Malignant transformation of hepatic adenoma complicated by rupture and hemorrhage: An extremely rare clinical entity
}

\author{
Rishi Philip Mathew ${ }^{1, *}$, Florin Manolea ${ }^{1}$, Safwat Girgis ${ }^{2}$, Vimal Patel ${ }^{1}$, Gavin Low ${ }^{1}$ \\ ${ }^{1}$ Department of Radiology \& Diagnostic Imaging, University of Alberta Hospital, Edmonton, Canada; \\ ${ }^{2}$ Department of Laboratory Medicine \& Pathology, Faculty of Medicine and Dentistry, University of Alberta Hospital, Edmonton, \\ Canada.
}

\begin{abstract}
Summary Hepatic adenomas (HAs) are rare benign tumors of the liver and comprise $2 \%$ of all liver tumors with an annual incidence of 3-4/100,000 per year in Europe and North America. These tumors may be clinically silent or present with abdominal pain. Although rare, the most important complications associated with this tumor is haemorrhage and malignant transformation to hepatocellular carcinoma. The reported risk of malignant transformation is believed to be $4.2 \%$. We present an extremely rare case report of a young woman on the oral contraceptive pill (OCP) with malignant transformation of a hepatic adenoma complicated additionally by tumor rupture and intraperitoneal bleed. This article therefore highlights the need to carefully evaluate any liver lesion in a young female on the OCP to be a possible adenoma and if confirmed to be so, to consider the potential risks associated with it as well as the need for follow-up imaging in order to avoid life threatening complications.
\end{abstract}

Keywords: Hepatic adenoma, inflammatory adenoma, hepatocellular carcinoma, oral contraceptive pill, hemorrhage, malignant transformation

\section{Introduction}

Hepatic adenomas (HAs) are rare benign tumors of the liver resulting from monoclonal proliferation of liver cells and comprise $2 \%$ of all liver tumors with an annual incidence of 3-4/100,000 per year in Europe and North America. Risk factors for the development and progression of HAs include- use of estrogen containing contraceptive pills, androgen or anabolic steroid intake (used primarily for management of Fanconi syndrome, impotence, body building and in transsexuals), conditions leading to impaired glycogenesis and excessive hepatic intracellular glycogen deposits such as glycogen storage diseases (GSD), familial adenomatous polyposis and metabolic syndromes like diabetes mellitus, insulin resistance, dyslipidemia and high blood pressure (1). Two recognized complications for HAs are hemorrhage and malignant transformation

\footnotetext{
*Address correspondence to:

Dr. Rishi Philip Mathew, Department of Radiology \& Diagnostic Imaging, University of Alberta Hospital, Suite-407, 8409112 St. NW, Edmonton, AB T6G 1K6, Canada.

E-mail:dr_rishimathew@yahoo.com
}

to hepatocellular carcinoma (HCC). It has been observed that HAs less than $5 \mathrm{~cm}$ tend not to bleed, while the reported risk of a malignant transformation of an HA to HCC is very low (4-5\%), and the reported mean size of solitary HA with features of malignant transformation is $10.5 \mathrm{~cm}$ (range: $4.5-18 \mathrm{~cm}$ ) (2).

We present a unique case of a solitary HA in a young woman on the oral contraceptive pill (OCP) that underwent malignant transformation, which subsequently ruptured and presented with intraabdominal hemorrhage. An extensive online search failed to reveal a similar documented case with both combinations of complications, and we felt our case report would be valuable for the worldwide medical fraternity.

\section{Case Report}

A young female in her mid-30s on the oral contraceptive pill presented with acute onset of abdominal pain, early satiety and indigestion. She had no relevant past or family history. On physical examination the patient appeared anemic. Her blood work revealed very low hemoglobin (70 g/L), elevated Alkaline Phosphatase (160 U/L) and elevated Alkaline Transferase (629 U/ 
L) and normal Total bilirubin (17 umol/L). She was referred for ultrasound examination of the abdomen for further evaluation.

Ultrasound of the abdomen revealed a $10 \mathrm{~cm}$ poorly defined hypoechoic lesion in the right lobe of the liver close to the capsule (Figure 1A). No significant vascularity was noted within the lesion on colour doppler. Additionally, turbid free fluid was noted in the pelvis in the right lower quadrant and in the posterior cul-de-sac (Figure 1B) raising the possibility of intrabdominal hemorrhage. The patient was immediately referred to our University Hospital for further evaluation and management.

The patient was further evaluated by contrast enhanced abdominal and pelvic computed tomography (CT). On non-contrast CT (Figure 2A), the liver appeared non-cirrhotic and demonstrated a $10.4 \times 9.5$ $\times 10.7 \mathrm{~cm}$ predominantly hyperdense lesion occupying majority of the right lobe of the liver. In addition, subcapsular hematoma involving the right lobe of the liver was noted, as well as adjacent intraperitoneal hemorrhage into the right subphrenic space with extension into the peritoneal cavity, with significant hemoperitoneum in the right lower quadrant and pelvis. On the post contrast image (Figure 2B), the lesion was noted to involve the entire right lobe (segments 5 to 8 ) and portions of segment $4 \mathrm{a}$ of the liver. In addition, peripheral areas of soft tissue enhancement were demonstrated in a patchy distribution. No additional lesions were noted in the liver. No intra or extra-hepatic vessel occlusion were seen. With the combination of these findings on $\mathrm{CT}$ along with the background history of OCP usage, the possibility of a ruptured adenoma was raised.

The patient was taken up for immediate surgery and an extended right hepatectomy including portions
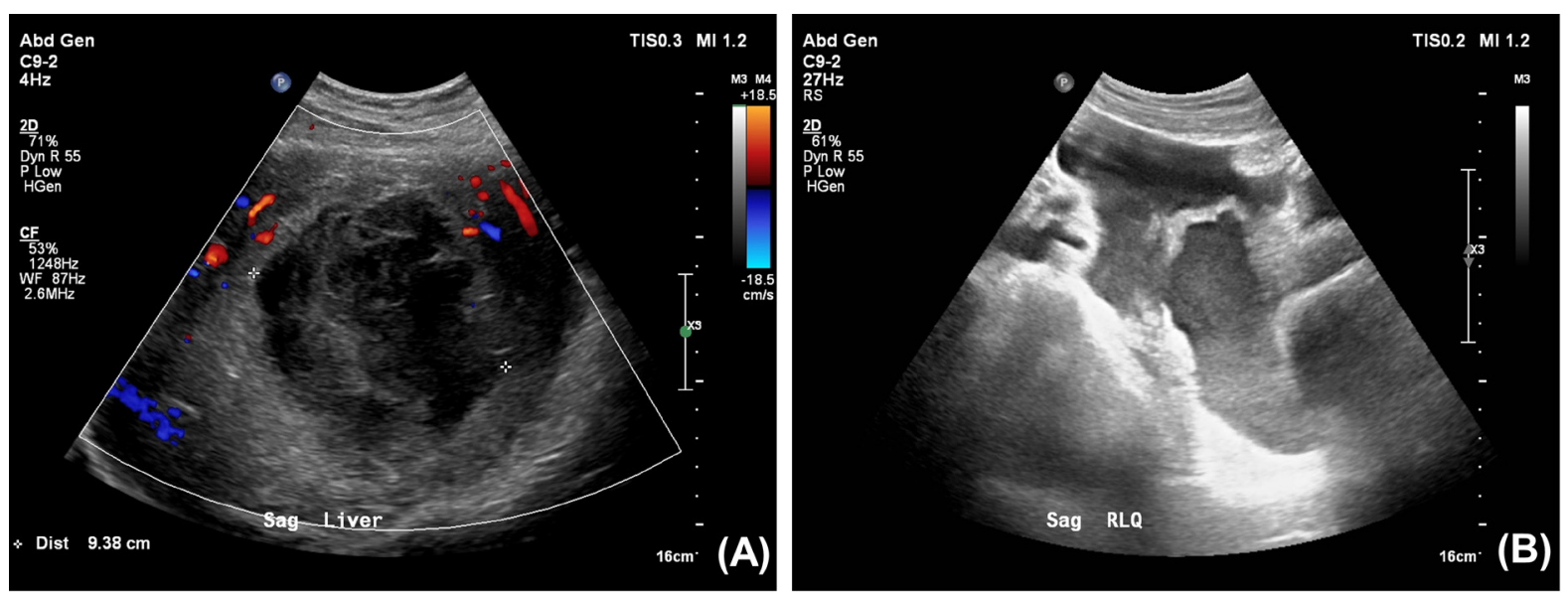

Figure 1. Ultrasound of the abdomen revealed a poorly defined non-vascular hypoechoic lesion (A) in the right lobe of the liver close to the liver capsule. Additionally, turbid free fluid was noted in the pelvis in the right lower quadrant (B) in the posterior cul-de-sac raising the possibility of intrabdominal hemorrhage.
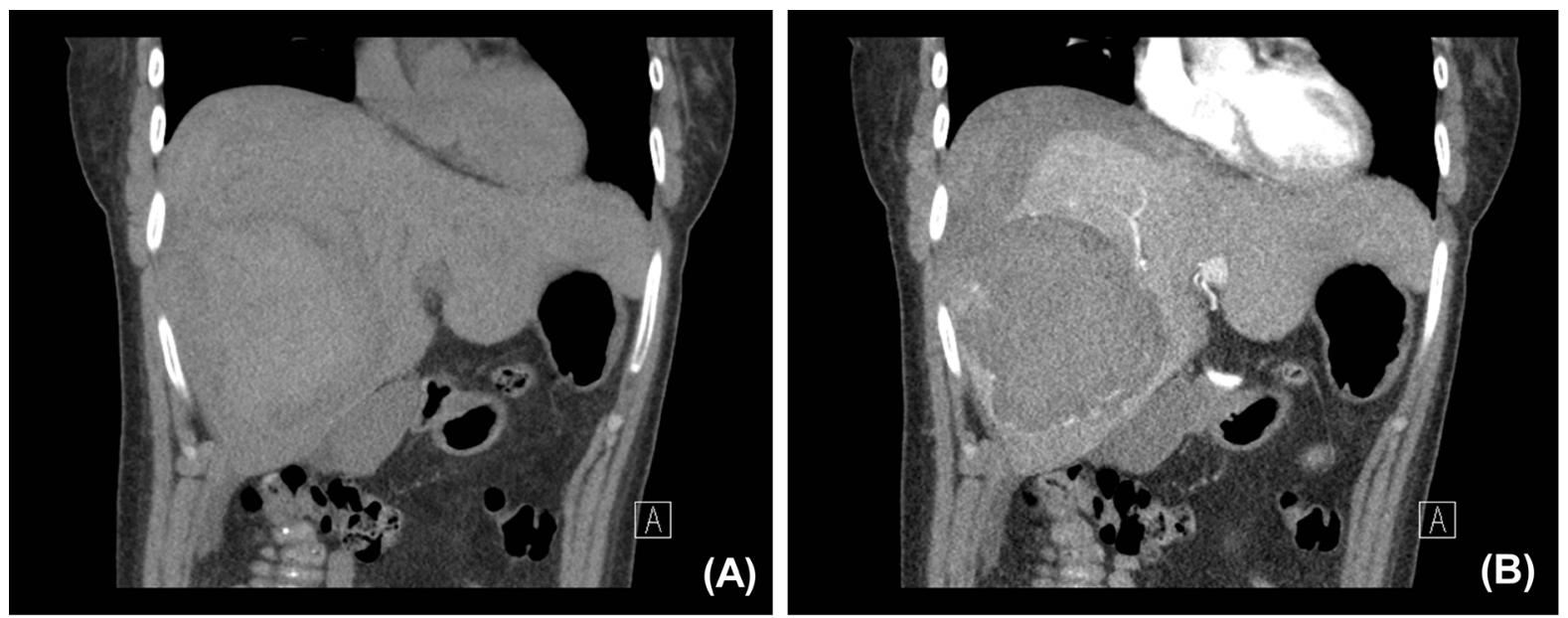

Figure 2. Non-contrast coronal reformatted CT image (A), showing non-cirrhotic liver with a $10 \mathrm{~cm}$ hyperdense lesion occupying the majority of the right lobe of the liver, in addition, subcapsular hematoma involving the right lobe of the liver as well as adjacent intraperitoneal hemorrhage into the right subphrenic space as well as into the peritoneal cavity was also identified. Post contrast coronal reformatted CT image (B) showing peripheral areas of soft tissue enhancement were demonstrated in a patchy distribution. In addition, a liver capsule defect was clearly visible, confirming lesion rupture and subsequent intraperitoneal hemorrhage. 

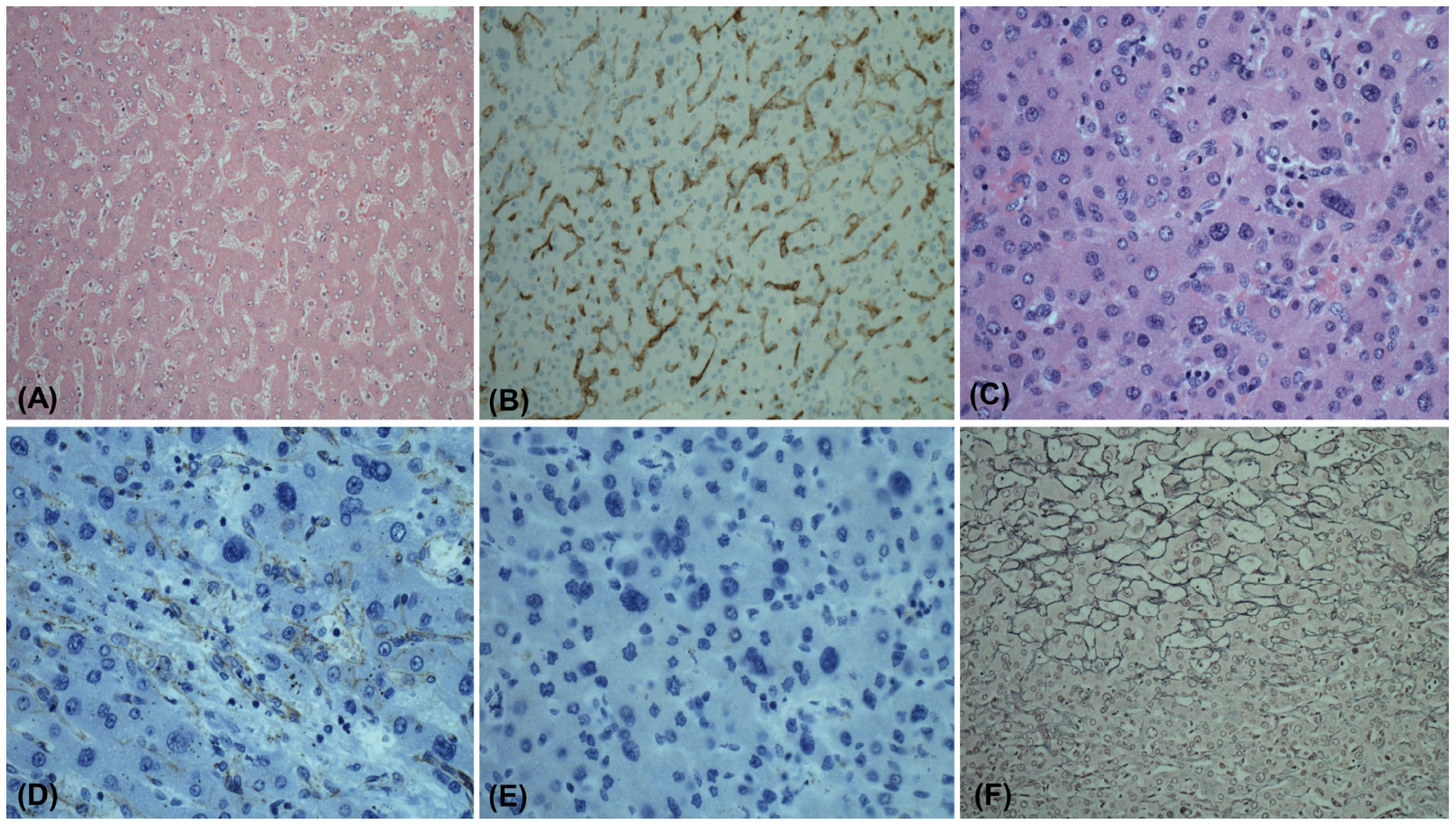

Figure 3. The adenoma at $100 \times(\mathbf{A})$, section shows liver parenchyma with no intervening portal tracts and normal trabecular pattern, one to two cell width. The HCC $200 \times(\mathbf{B})$, section shows liver parenchyma with visible marked atypia and patchy expanded trabeculae. Retic interface (C), section shows preservation of normal reticulin pattern in the adenomatous area while the malignant area shows reticulin loss. CD34 (D), sections positive expression of CD34 indicative of arterialization of the sinusoids. Negative Glypican3 (E), section shows malignant area with no expression of Glypican3. Negative Beta Catenin (F), sections shows malignant area with no expression of Beta Catenin.

of segment IVa and cholecystectomy was carried out. A final diagnosis of a ruptured poorly differentiated hepatocellular carcinoma arising within a hepatic adenoma was reached on pathological analysis of the resected specimen of the liver (Figure 3A-F).

The patient's postoperative course was unremarkable. She was advised to discontinue OCP usage.

Post-operative follow-up CT done 1.5 months later revealed no residual or recurrent tumor in the liver. A 9 $\times 5.3 \mathrm{~cm}$ fluid attenuating lesion was noted at the site of the hepatectomy margin, consistent with a post-operative seroma. In addition, compensatory hypertrophy of the left lobe of the liver was also noted. Post-operative alpha fetoprotein (AFP) was normal.

\section{Discussion}

HAs usually tend to be solitary (70-80\%) and less commonly multiple (20-30\%) (3). The term hepatic adenomatosis may be used by some authors when there are ten or more adenomas, and some in the past even considered hepatic adenomatosis to be a separate clinical and histological distinct entity from solitary HAs and multiple HAs $(<10)(4)$. Nevertheless, in recent years the term has lost its individuality and recent molecular classification has shown that the term HA comprises both solitary and multiple adenomas $(<10$ and $\geq 10)(5)$. Complications with HAs tend to occur when the tumors outgrow their blood supply and include rupture, hemorrhage, thrombosis, infarction, malignant transformation and rarely even cystic degeneration (6).

HAs can be classified into six major molecular subgroups namely: HNF $1 \alpha$ inactivated HA, inflammatory HA, b-catenin exon 3 mutated HA, b-catenin exon 7/8 mutated HA, Sonic Hedgehog activated HA and unclassified HA. The b-catenin exon 3 mutated HAs have a greater propensity for malignant transformation, while the Sonic Hedgehog activated HAs are at risk for bleeding (7). The Bordeaux group (8), identified useful immunohistochemical markers for identifying HA subtypes, namely, $\beta$-catenin and glutamine synthetase (GS) for $\beta$-catenin activated HA, liver-fatty acid binding protein (LFABP) for HNF$1 \alpha$ inactivated adenomas, serum amyloid A (SAA) and $\mathrm{C}$ - reactive protein (CRP) for the inflammatory subtype of HA. However, no markers were identified for the unclassified subtype. Risk factors for malignant transformation of HAs include- large size $(>5 \mathrm{~cm})$, multiplicity, $\beta$-catenin mutated subtype of HA and the male gender (9).

The commonly used imaging modalities for evaluation of HAs are ultrasound, contrast enhanced $\mathrm{CT}$ and magnetic resonance imaging (MRI). Typically, HAs $(<3-5 \mathrm{~cm}$ in size) on ultrasound appear isoechoic to background liver parenchyma, but these tumors can appear hypoechoic in patients with steatosis. However, HAs containing glycogen (e.g. in patients with GSD) or fat such as in HNF-1 $\alpha$ subtype, generally appear hyperechoic. Large HAs $(>5 \mathrm{~cm})$ tend to be 
Table 1. Magnetic resonance imaging (MRI) features of various hepatic adenoma (HA) subtypes

\begin{tabular}{|c|c|}
\hline HA Subtype & MRI Features \\
\hline Inflammatory HA & $\begin{array}{l}\text { - } \quad \text { T1 hypointense and T2 hyperintense } \\
\text { On out-of-phase T1 sequence: may occasionally demonstrate heterogenous loss of signal intensity because } \\
\text { of intralesional microscopic fat. } \\
\text { - Demonstrate a rim of higher T2 signal at the periphery than the centre of the lesion, termed the 'atoll sign'. } \\
\text { On dynamic imaging appears as a hypervascular mass with hyperenhancement during the arterial phase and } \\
\text { persisting hyperenhancement or isoenhancement on the portal and delayed phases. } \\
\text { On hepatobiliary phase: peripheral hyperintensity with central low signal intensity may be seen reflecting } \\
\text { the abnormal ductal dilatation and altered biliary excretion. }\end{array}$ \\
\hline HNF- $1 \alpha$-Mutated HAs & $\begin{array}{l}\text { - } \quad \text { T1 hyperintense and T2 iso-hypointense } \\
\text { On out-of-phase sequence: may demonstrate diffuse areas of loss of signal intensity because of intralesional } \\
\text { microscopic fat. } \\
\text { - On dynamic imaging: appears as a hypervascular mass but to a lesser extent than inflammatory HCAs, and } \\
\text { may become hypointense (washout) to liver parenchyma on the portal venous and delayed phases. } \\
\text { On hepatobiliary phase: appears homogenously hypointense in almost all cases. }\end{array}$ \\
\hline$\beta$-Catenin-Mutated HAs & $\begin{array}{l}\text { - } \quad \text { T1 heterogeneously hypointense and T2 heterogeneously hyperintense } \\
\text { On out-of-phase sequence: may demonstrate heterogenous areas of loss of signal intensity because of } \\
\text { intralesional microscopic fat. } \\
\text { On dynamic imaging appears as a homogenous or heterogenous hypervascular mass with persistent or non- } \\
\text { persistent enhancement during the delayed phase. } \\
\text { On hepatobiliary phase: can appear hypointense or hyperintense. }\end{array}$ \\
\hline Unclassified HAs. & As imaging experience is limited with this subtype no specific MRI pattern has yet been proposed. \\
\hline
\end{tabular}

heterogenous in appearance because hemorrhage or necrosis. Colour doppler has poor sensitivity and may demonstrate predominantly peripheral vascularity (10). On non-contrast CT, HAs appear as well circumscribed hypodense lesions (because of intra-tumoral fat, chronic hemorrhage or necrosis). In cases of acute hemorrhage, the tumor appears hyperdense. Calcifications may be seen in $5-10 \%$ of the cases. On post intravenous contrast administration these tumors show enhancement in the arterial phase becoming isodense to background liver parenchyma on the portal venous and delayed phases, although some lesions can show washout on the portal or delayed phases (11). MRI features of HAs can vary depending on the subtype and has been elaborated in detail in Table $1(3,12)$.

Management strategies for HAs depend on the underlying risk factors and size. In patients on hormone replacement therapies (including estrogen and androgen) complete cessation is recommended as studies have shown a regression rate of almost $80 \%$ and even complete resolution of the tumor in some patients on stopping OCPs (13). In obese patients, weight loss and even bariatric surgery has proved to be beneficial. For patients with GSD, alterations in dietary habits can cause tumor regression. HAs $<5 \mathrm{~cm}$ in size are managed conservatively as they tend to have a benign and uncomplicated clinical course and hence are followed up every 6 months by CT/MRI for the first two years and then annually. In hemodynamically stable patients with bleeding HAs, transarterial embolization (TAE) is considered as the first treatment option, with studies showing a $75 \%$ tumor regression rate (13). Limited available data has shown that ablative techniques (e.g. microwave ablation, percutaneous irreversible electroporation, and thermal ablation) are efficacious for small adenomas $(<5 \mathrm{~cm})$ especially in patients with comorbidities. As per current guidelines, surgical resection is recommended for HAs $>5 \mathrm{~cm}$ in size and those adenomas that don't regress or increases in size following OCP cessation during the 6-month followup interval (13). Surgery is also recommended for HAs demonstrating increase in size, belonging to the $\beta$-catenin subtype, rising alpha fetoprotein (AFP) levels, those with features of malignant transformation and HAs in males, as the latter are prone to have the $\beta$-catenin subtype. The main indications for liver transplantation includemultiple HAs not amenable to surgical resection with suspicious or confirmed malignant transformation, and the presence of portosystemic venous shunt (13).

\section{Conclusion}

Malignant transformation of HAs is proven and occurs in $4-5 \%$ of all cases. Our case is unique, as the HA not only underwent malignant transformation but was further complicated by rupture and intra-abdominal bleed. By identifying the risk factors for HAs and recognizing the imaging characteristics, an early and accurate diagnosis can be reached thereby enabling urgent intervention which could potentially be lifesaving.

\section{References}

1. Vijay A, Elaffandi A, Khalaf H. Hepatocellular adenoma: An update. World J Hepatol. 2015; 7:2603-2609.

2. Stoot JH, Coelen RJ, De Jong MC, Dejong CH. 
Malignant transformation of hepatocellular adenomas into hepatocellular carcinomas: A systematic review including more than 1600 adenoma cases. HPB (Oxford). 2010; 12:509-522.

3. Grazioli L, Olivetti L, Mazza G, Bondioni MP. MR imaging of hepatocellular adenomas and differential diagnosis dilemma. Int J Hepatol. 2013; 2013:374170.

4. Flejou JF, Barge J, Menu Y, Degott C, Bismuth H, Potet F, Benhamou JP. Liver adenomatosis. An entity distinct from liver adenoma? Gastroenterology. 1985; 89:11321138.

5. Frulio N, Chiche L, Bioulac-Sage P, Balabaud C. Hepatocellular adenomatosis: What should the term stand for! Clin Res Hepatol Gastroenterol. 2014; 38:132-136.

6. Foo SY, Paul L, Viswanathan S. Cystic degeneration of hepatic adenoma: A rare complication of hepatic adenoma. BJR Case Rep. 2017; 4:20170056.

7. Nault JC, Paradis V, Cherqui D, Vilgrain V, ZucmanRossi J. Molecular classification of hepatocellular adenoma in clinical practice. J Hepatol. 2017; 67:10741083.

8. Bioulac-Sage P, Rebouissou S, Thomas C, Blanc JF, Saric J, Sa Cunha A, Rullier A, Cubel G, Couchy G, Imbeaud S, Balabaud C, Zucman-Rossi J. Hepatocellular adenoma subtype classification using molecular markers and immunohistochemistry. Hepatology. 2007; 46:740748.

9. Farges O, Dokmak S. Malignant transformation of liver adenoma: An analysis of the literature. Dig Surg. 2010; 27:32-38.

10. Dietrich CF, Tannapfel A, Jang HJ, Kim TK, Burns PN, Dong Y. Ultrasound imaging of hepatocellular adenoma using the new histology classification. Ultrasound Med Biol. 2019; 45:1-10.

11. Faria SC, Iyer RB, Rashid A, Whitman GJ. Hepatic adenoma. AJR Am J Roentgenol. 2004; 182:1520.

12. Wang H, Yang C, Rao S, Ji Y, Han J, Sheng R, Zeng M. MR imaging of hepatocellular adenomas on genotypephenotype classification: A report from China. Eur J Radiol. 2018; 100:135-141.

13. Tsilimigras DI, Rahnemai-Azar AA, NtanasisStathopoulos I, Gavriatopoulou M, Moris D, Spartalis E, Cloyd JM, Weber SM, Pawlik TM. Current approaches in the management of hepatic adenomas. J Gastrointest Surg. 2019; 23:199-209.

(Received August 3, 2019; Revised November 25, 2019; Accepted November 28, 2019) 